This document is confidential and is proprietary to the American Chemical Society and its authors. Do not copy or disclose without written permission. If you have received this item in error, notify the sender and delete all copies.

\title{
GlyConnect: glycoproteomics goes visual, interactive and analytical
}

\begin{tabular}{|r|l|}
\hline Journal: & Journal of Proteome Research \\
\hline Manuscript ID & pr-2018-007668.R1 \\
\hline Date Submitted by the \\
Author: & n/a \\
\hline Complete List of Authors: & $\begin{array}{l}\text { Alocci, Davide; Swiss Institute of Bioinformatics, Proteome Informatics } \\
\text { Group; Universite de Geneve Centre Universitaire d } \backslash \text { Informatique, } \\
\text { Computer Science Department CUI } \\
\text { Mariethoz, Julien; Swiss Institute of Bioinformatics, Proteome } \\
\text { Informatics Group } \\
\text { Gastaldello, Alessandra; Swiss Institute of Bioinformatics, Proteome } \\
\text { Informatics Group } \\
\text { Gasteiger, Elisabeth; SIB Swiss Institute of Bioinformatics } \\
\text { Karlsson, Niclas; University of Gothenburg, The Sahlgrenska Academy, } \\
\text { Medical Biochemistry } \\
\text { Kolarich, Daniel; Institute for Glycomics, Griffith University } \\
\text { Packer, Nicolle; Macquarie University, Department of Chemistry and } \\
\text { Biomolecular Sciences } \\
\text { Lisacek, Frederique; Swiss Institute of Bioinformatics, Proteome } \\
\text { Informatics Group }\end{array}$ \\
\hline
\end{tabular}

\section{SCHOLARONE" Manuscripts}




\title{
GlyConnect: glycoproteomics goes visual, interactive
}

\section{and analytical}

Davide Alocci ${ }^{1,2}$, Julien Mariethoz ${ }^{1,2}$, Alessandra Gastaldello ${ }^{1,2}$, Elisabeth Gasteiger ${ }^{3}$, Niclas G Karlsson ${ }^{4}$, Daniel Kolarich ${ }^{5,6}$, Nicolle H Packer ${ }^{5,6,7}$, Frédérique Lisacek ${ }^{1,2,8}$

1. Proteome Informatics Group, SIB Swiss Institute of Bioinformatics, Rue Michel-Servet 1, CH-1211 Geneva, Switzerland

2. Computer Science Department, University of Geneva, Switzerland

3. Swiss-Prot Group, SIB Swiss Institute of Bioinformatics, Geneva, Switzerland

4. Department of Medical Biochemistry and Cell Biology, Institute of Biomedicine, University of Gothenburg, Sweden

5. Institute for Glycomics, Griffith University, Southport, Queensland, Australia

6. ARC Centre for Nanoscale BioPhotonics, Macquarie University and Griffith University, Australia

7. Department of Molecular Sciences, Macquarie University, Sydney, NSW, Australia

8. Section of Biology, University of Geneva, Switzerland

Keywords: Data integration, database, software, glycoprotein, glycomics, glycoproteomics, glycan structure, mass spectrometry, data curation, data visualisation

\begin{abstract}
Knowledge of glycoproteins, their site-specific glycosylation patterns and the glycan structures that they present to their recognition partners in health and disease are gradually being built on using a range of experimental approaches. The data from these analyses are increasingly being standardised and presented in various sources, from supplemental tables in publications to localised servers in investigator laboratories. Bioinformatics tools are now needed to collect this data and enable the user to search, display and connect glycomics and glycoproteomics to other sources of related proteomics, genomics and interactomics information. We here introduce GlyConnect (https://glyconnect.expasy.org/), the central platform of the Glycomics@ExPASy portal for glycoinformatics. GlyConnect has been developed to gather, monitor, integrate and visualize data in a user-friendly way in order to facilitate the interpretation of collected glycoscience data. GlyConnect is designed to accommodate and integrate multiple data types as they are increasingly produced.
\end{abstract}




\section{Introduction}

The importance of identifying proteoforms is increasingly stressed in the proteomics community and the intensive work involved in assessing their variety and extent was recently discussed ${ }^{1}$. In that overview, the case of glycoforms was singled out and the contribution of glycoproteomics discussed. The heterogeneity of glycoproteins and the variety of techniques that are used for their study has raised obstacles to the automation of the analytical procedures. Progress in this area has also been limited by the lack of standardised, open access software for integrating glycomics with other -omics data. A current consensus regarding advances in glycosciences is the acknowledgement of the bottleneck created by limited bioinformatics support.

The broad range of databases and tools that can support functional studies of mammalian glycoproteins includes glycan structure collections, mass spectrometry and chromatographic analysis tools, glycoenzyme classification, glycoprotein 3D structure as well as glycan and lectin array interaction databases (see overview of current resources in recent reviews ${ }^{2} 34$ ). These have all been developed since the landmark glycoinformatics efforts were begun with CarbBank ${ }^{5}$ and first connected to other resources in glycobiology by GLYCOSCIENCES. de ${ }^{6}$ and the Consortium for Functional Glycomics $(\mathrm{CFG})^{7}$. A few years ago, we initiated the development of interactive and exploratory tools to populate the Glycomics@ExPASy portal ${ }^{8}$. As the next step, we introduce here GlyConnect, a central platform integrating glycoproteomic data and related software in a user-friendly resource. By design, GlyConnect will enable its use by all biologists investigating biomolecular functions. In particular, emphasis is put on usability and data visualisation.

Despite its importance, data visualisation remains a challenge in glycobiology. The adoption of a cartoon notation ${ }^{10}$ simplifying the representation of glycans was a major step in spreading interest in these molecules and easing their use in databases and software. Nonetheless, only a handful of visualisation tools have improved some aspects of glycan identification and structural representation. Glycoviewer ${ }^{11}$ is the first example of a tool that allows glycoscientists to visualise, summarise and compare different glycomes. GlycomeAtlas $^{12}$ provides an interactive interface for exploring CFG array data. In parallel, three-dimensional modelling has also generated interactive images of glyco-molecules ${ }^{13}$ and 
glyconjugates ${ }^{14}$ as recently reviewed ${ }^{15}$. GlycoDomainViewer ${ }^{16}$ was introduced as a visual "integrative tool for glycoproteomics that enables global analysis of the interplay between protein sequences, glycosites, types of glycosylation, and local protein fold /domain and other PTM contexts". GlycoDomainViewer integrates experimental data as well as knowledge data sources presenting an extensive collection of information to explore the possible effect of glycosylation on a protein.

To enrich this collection and build custom visualisation, we have combined different gateways into glycoknowledge. Glycobiologists start from their knowledge about glycans and correlate, for example, proteins or disease, whereas proteomics experts focus on the glycosylation of a protein(s) of interest. GlyConnect presents a series of interactive diagrams that help the user understand relations between glycans, proteins, tissues, diseases and taxonomy. Search results are presented in a conceptual map, which shows the connections between glycans and proteins via glycan compositions. User-friendly visualisation allows nonexpert scientists to explore the data and break the barrier between different disciplines. We bridge glycomics with proteomics by cross-referencing each protein with UniProt ${ }^{17}$ as well as neXtProt ${ }^{18}$, a knowledge platform for human proteins. We have also associated each glycoprotein with GeneCards ${ }^{19}$, a searchable and integrative database that compiles information on all annotated and predicted human genes. We have initiated a series of "Glycome" pages that summarise all the information available for the glycome specific for a tissue or body fluid. The first page has been focusing on the human plasma $\mathrm{N}$-glycome and is available from the main menu under "Glycome".

The present article highlights the features of GlyConnect and through examples of data searches, describes the potential of the platform for revealing unexpected associations as well as formulating new questions regarding glycosylated proteins.

\section{Material and Methods}

\section{Data}

The molecular entities that are described in the database are shown in Figure 1. 


\section{Database content}

GlyConnect data content spans information on glycan structures and compositions, glycoproteins, glycosylation sites, taxonomy, tissue expression and diseases. Approximately $70 \%$ of the data in GlyConnect is manually curated. It includes the full content of the GlycoSuiteDB database ${ }^{20}$ licensed by SIB with additional curated data by Robyn Peterson (former Packer lab member). This information is labelled in the database as reviewed. It also contains data extracted from the supplementary material of several large-scale glycoproteomics studies recently published ${ }^{21}{ }^{22}$. This supplementary material was downloaded and partially curated only to reduce redundancy and enhance the consistency of the database. For example, when several accession numbers are listed for a single protein, these are reviewed and merged whenever possible. A cross-link to UniProtKB/Swiss-Prot is always favoured ahead of one to UniProtKB/TrEMBL. Additional filters such as the minimum size of a peptide (at least seven amino acid-long) validating a glycosite, are also applied. This data has introduced a bias towards a majority of human glycoproteins in the current version of the database.

\section{Cross-references}

Information in the database is supported with a broad range of cross-references. Table I summarises them all, with corresponding URLs.

\section{Database model}

The data scheme is inherited from the GlycoSuiteDB database. The database content was transferred from Proteome Systems Ltd to SIB Swiss Institute of Bioinformatics under a license agreement in 2009. This content was structured in a spreadsheet-like manner with a single table. To manage this non-normalised and error-prone architecture, we applied the current and usual database normalisation techniques ${ }^{23}$. This normalisation process helped to target and remove errors and duplications. The concept of normalisation consists mostly in splitting data tables into smaller tables to reduce redundancy and improve data integrity. We applied data modelling techniques to guarantee the uniqueness of glycan structures, compositions, proteins, expression tissues, diseases and references supporting the information. This normalisation process was applied to preserve the relatedness of information established in publications and ease navigation between entities (glycan, protein, expression tissue, disease, 
etc.) by keeping contextual information. The redesigned database now complies with the Essential Tuple Normal Form (ETNF) and as such, prevents tuple redundancy as well as observes at least the four first normal forms of the relational model ${ }^{24}$. This now makes the refactored dataset robust, consistent and easier to extend. The latter was verified when adding the large-scale glycoproteomics data. This process, which entailed integrating new concepts like peptide and proteoform while fitting the existing data model, was rapidly implemented and applied.

While GlyConnect is a glycoprotein-centric database, glycosylation still remains the core concept inherited from its ancestor. The glycosylation notion of the database is built using the association of a glycan structure with a given protein at a specific amino acid position. Moreover, the glycosylation is supported by one or more published articles and a given article may relate the glycosylation to a disease. The conceptualisation and entity relationships reflect the knowledge of the glycobiology.

In addition to the relational data model, an RDF (Resource Description Framework) version of the GlyConnect data is under construction. This process relies on a glycoconjugate ontology currently under development. Providing an RDF version of the GlyConnect data will support federated queries with UniProt ${ }^{17}$, nextProt ${ }^{18}$, GlyTouCan ${ }^{25}$ or any other triplestore of interest. This leads to an additional level of integrability using RDF and semantic web technology.

\section{Encoding and display of glycans}

Many formats have been created to encode and represent glycan structures ${ }^{26}$. In GlyConnect we have selected the current most popular ones in glycoinformatics. Glycan structures are stored in the GlycoCT format ${ }^{27}$. They are displayed by default with the SNFG notation ${ }^{9}$ promoted in the reference manual Essentials of Glycobiology ${ }^{28}$. This cartoon representation of glycans simplifies the otherwise dense atomic description. Structures can also be viewed in the IUPAC condensed ${ }^{29}$ and the oxford $^{30}$ formats.

\section{Design and Implementation}

GlyConnect is intended as a dashboard to monitor and facilitate the interpretation of glycomics and glycoproteomics data. Its design reflects this aim. Users can access GlyConnect from different directions either through a web interface or using the Application 
Programming Interface (API). GlyConnect data are stored using PostgreSQL 9.5, and they are part of the Glycomics@ExPASy initiative ${ }^{8}$. Additionally, GlyConnect has full access to an internal glyco-epitope database and to SugarBindDB ${ }^{31}$, a database of pathogen-glycan interactions. The API is built with the open source framework Play (version 2.5) that combines Java and Scala code and allows the separation between model, view and controller. In GlyConnect the model is represented by the mapping class built with Ebean, an objectrelational mapping (ORM) which converts each table row from the database into a java object. Controllers link models and views and provide API calls.

The central element of GlyConnect is the database that contains both reviewed and unreviewed data. As shown in Figure 2, there are two main types of access: Search and Browse. The web interface of GlyConnect is a mixture of technologies. The Search Interface (left frame of Figure 2) available under the "Search" tab has been developed using web components and in particular the library Google Polymer (Version 2). Web components are reusable user interface widgets, which are built using a mixture of HTML5, CSS3 and Javascript. In GlyConnect we have used Google Polymer, which speeds up the development of custom web components fulfilling the standard established by the W3C. The Browse Interface (right frame of Figure 2) available under the "Browse" tab is built with Play as stated above. Pages displayed in that section are views generated from the database built with Scala and include Twitter Bootstrap as well as several other Javascript libraries to improve userfriendliness.

\section{Search interface}

The search interface is the main entry point to query data available in the GlyConnect database. As hinted in its name, GlyConnect outputs relationships between glycans and proteins or glycans and tissues or taxonomy. Users can search either proteins or glycans. Starting with proteins is rather straightforward while querying with glycans offers several options. Since glycan structures are notoriously heterogeneous, the search interface allows the user to query by monosaccharide composition, by structural features (such as fucosylation or sialylation) or by structural motif. 


\section{Search by protein}

A protein name or UniProt accession number available in the database can be selected from a dropdown list. Since analogous proteins can be found in multiple species, a box displays all choices of available species. After selecting the species, clicking on the search button will perform the query.

\section{Search by monosaccharide composition}

Glycan compositions can be input manually or uploaded in a file. Alternatively, the "Monosaccharide selectors" tab provides a wizard to define compositions in an acceptable format. The three options are available in three successive tabs. Clicking on the search button launches the query.

\section{Search by linkage type}

The database queries reflect the classical grouping of glycans based on the distinct types of attachment to proteins: $\mathrm{N}$-Linked, $\mathrm{O}$-Linked and free glycans (released from conjugates). The interface proposes three levels of selection of structural features organised in three successive tabs. In the first tab, the broad classification of $N$-linked (oligomannose, complex, etc) or $O$-linked (core 1,2,3, etc) is provided in the form of selectable items to be dropped in the query box. In the second tab, a series of more distinct properties is available for selection with the same principle of dropping items into the query box. A complete list of all recorded features is given in Table Ila,b. In the interface, the colour code for each box/feature matches that of the SNFG notation (red in relation to fucose, yellow in relation to galactose, etc). The selection, if any, of features in one tab remains active when performing a selection in another tab. In this way, the user can combine criteria and search, for example, oligomannose structures (first tab), core-fucoslyated (second tab), then make a choice in the third tab from a list of structural motifs (limited to known determinants, such as sialyl Lewis $\mathrm{x}$, blood group $B$, etc). In the third tab, an SNFG picture of each motif helps the user in selecting those of interest. Clicking on the search button launches the query. 


\section{Conceptual Map (aka octopus)}

The results of each query performed in the search interface are displayed as a conceptual map, the shape of which evokes an octopus. We use this image to describe the content. For the sake of clarity, we have used a compact notation for compositions that is presented in Table III. The centre of the map contains the list of monosaccharide compositions of the glycans. Information in the left and right parts of the map connected with "tentacles" to compositions can change according to the option selected in the toolbar above the map. Several possibilities are given to the user:

- Protein - Glycan

- Protein - Taxonomy

- Protein - Disease

- Disease - Glycan

- Tissue - Disease

- Tissue - Glycan

Each possibility reflects the values that will populate the map. For example, if Protein Taxonomy is selected, then protein names on the left side will be connected to corresponding taxa on the right side via monosaccharide compositions in the centre. Every time a new map option is selected, the octopus is updated: and the shape adjusted; only the central information on glycan compositions remains.

If the search criteria are broad, the map may be overpopulated. A message prompt is then displayed and invites the user to apply stricter criteria. This can be done by relaunching a search with additional criteria. We also included a quick fix with a series of filters located above the conceptual map. The taxonomy filter reduces the size of the octopus by restricting the results to taxa of interest. Two combo boxes compose the taxonomy filter, the first limits the search to taxonomy classes, e.g., Mammalia, whereas the second sets a specific species. The date filter restricts the results to data evidenced by articles published within a set time bracket.

The conceptual map represents the entry point to navigate query results. Mouse-hovering on a box, in the centre of the map, highlights the tentacles that have a connection with the selected composition. Those tentacles can be coloured in red by clicking on the box. A second 
click on the box restores the grey colour deselecting the tentacles. By clicking on each dot of the left and right sides, the user can obtain more details about a particular glycan, protein, taxonomy, disease or tissue. Protein and glycan pages share the same look of the search interface whereas all the other pages (Taxonomy, Disease, Tissue) redirect the user to the browse interface.

Since each extremity of a tentacle links to information that is either reviewed or unreviewed, different colours distinguish evidence checked by expert curators from evidence extracted from high-throughput studies. Light-blue has been assigned to reviewed contents while grey identifies unreviewed information. In the octopus, dots at the tip of tentacles and composition boxes in the central part change colours according to the status of the associated content.

Results obtained in GlyConnect can be forwarded to GlycoSiteAlign ${ }^{32}$ that aligns the amino acid sequences surrounding glycosites depending on the properties of the attached glycans. Clicking on "Align glycosites with GlycoSiteAlign" sends all the glycan information (compositions and structural features such as "fucoslyated") on glycosites of selected proteins to GlycoSiteAlign. This will result in displaying the amino acid sequence alignment (by default, 20 positions on each side of the glycosylated residue).

\section{Protein and Glycan pages}

Protein and glycan pages have been engineered to: (1) display relevant information stored in the database and (2) whenever possible, integrate tools developed as part of the Glycomics@ExPASy initiative. The overall display is similar for both glycans and proteins:

(a) A star preceding the name of the entity at the top of a page qualifies the status of the information contained in that page: a star filled in yellow qualifies reviewed information whereas an empty star identifies unreviewed data.

(b) Cross-references to external databases are listed at the top of a page

(c) A page is composed of several sections that are delineated with blue bars

(d) Sankey graphs are shown in a section labelled "Associations" to display the relationships between the glycoprotein or the glycan structure of interest and other 
entities of the database, namely, glycosites, tissues and diseases. These graphs are currently not hyperlinked to the database (see future work)

(e) PubMed references provided as supporting evidence are shown in a section labelled "References"; links to DOI are also included.

\section{Specificity of glycan pages}

In a glycan page, the different sections are arranged in tabs coloured in blue (resp. grey) when selected (resp. not selected). A specific tab labelled "Family" contains the result of a composition search across the database shown as a list of the possible arrangements of the same monosaccharide building blocks displayed as SNFG images. This list can in turn serve as input to EpitopeXtractor ${ }^{33}$. This tool will perform an extraction of all the known glycoepitopes, based on a curated list of almost 600 glycan determinants ${ }^{33}$. This input can be modified by the user as items of the list can be removed acting on a red icon next to each glycan image.

\section{Specificity of protein pages}

In a protein page, the different sections are arranged vertically with headers boxed in blue. A specific section of the page contains site-specific information and it is labelled "Sites". Each site is presented in a collapsible container featuring the glycosylated amino acid and its relative position on the protein (e.g. "Asn-234") unless evidence is partial and the site is unknown and labelled as such. The header is red (resp. grey) if the evidence is reviewed (resp. unreviewed). Once collapsed, glycans that were identified on that site are listed as SNFG images. In each site-specific section, defined structures as well as compositions are listed together. Since some compositions and structures may actually match one another, this relationship is captured by grouping the matching structure(s) and composition(s) within a single frame. Furthermore, glycan structures listed in the unknown site section are matched to reported compositions for possible site assignment. To this end, they are singled out with a grey border in the interface. When the information is extracted from mass spectrometry data, the corresponding list of identified glycopeptides is provided. Peptides are listed after the glycan images when this information is available. 
In a protein page, all SNFG images of listed glycan structures are linked to their corresponding glycan page.

\section{Browse Interface}

The Browse interface is the entry point to explore data available in the GlyConnect database. Each molecular entity of Figure 1 is accessible in the top menu (grey bar). A colour code is preserved throughout to distinguish each of the nine data types: taxonomy [red], protein [dark yellow], tissue [green], (glycan) structure [light blue], (glycan) composition [purple], disease [pink], (published) reference [dark blue], (glycosylation) site [brown], (glycosylated) peptide [orange]. Each the coloured boxes (except the last two) of the homepage as shown in Figure 2, links to the list of corresponding entries. Neither "Sites" nor "Peptides" are displayed as independent entities.

The interface is built to facilitate navigation from one entity to the other to support data exploration. For example, starting with the list of structures available either from the homepage by clicking on the "Structures" button or selecting "Structures" in the top menu (grey bar) and selecting a particular one by clicking on it will open a page with the details of that structure (SNFG image, IUPAC code, GlycoCT code, GlyTouCan ${ }^{25}$ cross-reference). Statistics are updated in the coloured boxes showing the number and the names of proteins, tissues, species, etc, where this structure is found along with the references supporting these relationships. Any of the pieces of information displayed is clickable and allow further exploration of protein/tissue/species/disease/site/peptide. With this framework, any entity is a potential starting point and contextual information can be collected through the navigation from one data type to the other.

Since the coverage of entities (proteins, glycans, etc) is ever-growing, filters are implemented to reduce the size of lists displayed on the screen. For example, proteins can be filtered by name or UniProt accession number and species by taxa. Note that this display contrasts with the Search interface, as lists of proteins can be handled simply by selecting appropriate terms in the filter area. 


\section{RESTful API}

The Play web application of GlyConnect has two main components. It supports the web pages of the Browse Interface as well as other data related services using a RESTful API (application program interface) architecture. The purpose of the API is mainly to feed the Search Interface and other frontends with json formatted responses. When a query is built from the search interface, the search parameters are sent to the API through a URL that in turn sends a json representation of the result. The search option parameters, the conceptual map, the glycan and protein pages and the Sankey graphs are built with json data. The role of the API is to forward data from the database to the service processing the data in a standard format that is easy to parse. Some other services are accessible through the API and used in external applications. Since a glycan structure is usually searched in the database using the GlycoCT format, the API provides services to convert glycan encoding formats. This includes a textual converter from IUPAC of glycan to GlycoCT and an image generator that uses GlycanBuilder libraries ${ }^{34}$ and takes GlycoCT codes to return a cartoon representation (SNFG, Oxford, IUPAC 2D) in an image file (PNG, SVG BMP, SVG) that can be saved. Another API service compiles a list of glyco-epitopes. A composition search is also available and used by Unimod $^{35}$ to redirect users to GlyConnect. Finally, the API provisions mappings of GlyConnect with UniProt, GeneCards ${ }^{19}$ and neXtProt to feed each respective data integration pipeline.

\section{Glycome Pages}

The search and browse interfaces of GlyConnect allow users to search a specific glycan/protein but it is impossible to visualise and compare multiple glycans/proteins at the same time. For this reason, we have initiated the generation of more integrated "Glycome" pages. Each of these pages is destined to focus on a specific glycoproteome by providing combined information on protein and glycan expression. The first glycome page is dedicated to the $\mathrm{N}$-glycome of the human plasma. All the data has been extracted from Clerc et al. ${ }^{36}$ that reviewed human plasma protein $\mathrm{N}$-glycosylation. In this article, the glycosylation of plasma proteins is put into perspective so that the contribution of individual proteins to a total plasma $\mathrm{N}$-glycosylation profile is examined at the released glycan level. The result of this work is stored in a large table that shows glycoproteins in relation to glycan species in the form of monosaccharide compositions. We transformed this static table into interactive 
diagrams linked to our database. This was achieved by creating ad hoc visualisation, as follows:

1) The "Protein Abundance" pane shows the relative abundance of the 24 plasma glycoproteins listed by Clerc et al. ${ }^{36}$ Each protein is associated with a coloured disk, and the dimension of the disk is proportional to the relative abundance of the protein in the plasma. The disks are displayed in a spiral clockwise from the largest to the smallest. Hovering over a disk shows the protein name. Next to the spiral, glycoproteins are shown by names and grouped by main biological processes as per their Gene Ontology terms collected in the corresponding UniProt entries. Clicking on a name in any of these groups opens the related protein page in GlyConnect (search interface) whereas clicking on a disk triggers a change in the "Glycan Abundance per Protein" pane.

2) The "Single glycan abundance" pane shows the relative abundance of a single glycan composition expressed on each of the 24 glycoproteins listed in the previous pane.

3) The "Glycan abundance per protein" pane illustrates the relative abundance of each glycan composition found on a selected glycoprotein. The relative abundance is visualised using a bar that can change colour according to the threshold defined by the user. This interface is built on the same principle as that of Glynsight ${ }^{33}$ where the dependencies between compositions (for example H4N4F1 contains H4N4 and the two differ by one fucose) are included in the visualisation of the glycome profile.

As stated before, to select a different protein simply involves clicking on a different disk in the "Protein Abundance" pane. Also, clicking on a bar in the "Glycan abundance per protein" pane will trigger a change in the "Single glycan abundance" and "Glycan structures" panes.

4) The "Glycan structures" pane shows all currently reported, possible glycan structures that match a specific monosaccharide composition retrieved from GlyConnect glycan structures. The "Glycan structures" pane changes its content according to the glycan composition selected in the "Glycan abundance per protein" pane. 


\section{Results}

\section{Integrated platform for glycobiology}

GlyConnect includes data extracted from a broad range of published articles ranging from the characterisation of the global glycome of glycans in a biological system, a handful of glycans attached to an individual protein or the full screening of a cell glycoproteome. The content of the database reflects the current bias found in the literature. At present, it is clearly leaning towards released glycan characterisation or glycoprotein site-specific characterisation of mammalian $\mathrm{N}$-glycan compositions. This is a direct consequence of the recent improvements made for handling high throughput mass spectrometry techniques in glycomics (the glycans released from proteins) and glycoproteomics (the analysis of intact glycopeptides). The current statistics are visible on the homepage of the Browser section. In addition to the GlyConnect core data, the database also integrates cross-references (see Table I) and tools for users to process the database content and build hypotheses based on previously unseen associations. As shown in Figure 3, GlyConnect data is accessible through two distinct interfaces; while the Browse interface simply displays information stored in the database (unless filtered), the Search Interface relies on the API to extract only the information relevant to the search criteria.

Figure 3 illustrates the overall connectivity of the resource. Focusing on the Search interface (Bottom of Figure 3), users can explore search results by clicking on glycans and proteins presented in the octopus. Besides showing all the information stored in the database, glycan and protein pages are bound to respectively LiteMol ${ }^{37}$ and EpitopeXtractor. When available, LiteMol shows a 3D model of the glycoprotein together with the glycan moieties using the SNFG 3D notation ${ }^{14}$. In complement, EpitopeXtractor is integrated into each glycan page to present all structures with the same composition as the glycan described in the page, and then performs a glycan epitope extraction. This tool checks which known ligands are present in a selected set of structures by relying on a curated reference set of almost 600 epitopes ${ }^{33}$. Additionally, the results of EpitopeXtractor can be overlaid on the glycoepitope network proposed by Glydin', the glycan epitope network viewer ${ }^{33}$. Finally, as mentioned in the Material and Methods section, a query can be directly forwarded to GlycoSiteAlign ${ }^{32}$ to perform a protein sequence alignment based on selected glycan traits. 
The glycan and protein pages in the Search and Browse interfaces share the same information in their respective pages, especially regarding cross-references. To begin with, each page contains the PubMed IDs and article summary details including the DOI when the publication information is available as supporting evidence for the glycan assignment. Furthermore, protein pages link to protein (UniProt, neXtProt for human) and gene databases (GeneCards for human) whereas glycan pages are cross-linked to GlyTouCan, the glycan structure repository. Through GlyTouCan, cross-talk between GlyConnect and the MS/MS spectral database, UniCarb-DB ${ }^{38}$ is enabled. Additionally, the Browser interface has a collection of other pages for taxonomy, tissue and disease (Top of Figure 3). Each of these page types shows specific cross-references to expert resources (NCBI taxonomy browser ${ }^{39}$, Brenda ${ }^{40}$ and Uberon $^{41}$ for standard tissue references and disease ontology ${ }^{42}$ ).

Although the Search and Browse interfaces show the same information, they are specifically designed to answer different questions. We can illustrate the specificity of the Search interface with a query that cannot be expressed in the Browse interface. For example, assume our aim is to find all proteins that carry $O$-linked glycan structures containing the Sialyl-GalNAc epitope which actually also is the motif of the Sialyl-Tn antigen in Homo sapiens, i.e., NeuAc( $\alpha 2-6)$-GalNAc. In the Search interface clicking on the $O$-linked button restricts the search only to $O$-linked glycans. Then, the Sialyl-Tn epitope is selected in the "Determinants" tab and the query is run. The composition H1N1S1 is the exact composition of core 1 or core 8 trisaccharide O-glycans which differs by one galactose from the Sialyl-Tn antigen motif/NeuAc-GalNAc. Consequently, the connections highlighted in red in the octopus show on the structure side, only core 1 and core 8 and on the protein side (left) 18 proteins that are connected, six of which are common with those shown for the Sialyl-Tn antigen motif/ NeuAcGalNAc. The resulting octopus shows connections between 50 reported compositions matching 83 O-linked glycan structures containing the Sialyl-Tn epitope and 96 proteins known to carry structures including this motif. Clicking on a composition highlights in red the relationship(s) between corresponding glycan structures and glycoproteins as reported in the database. Some relations are unique, and some are multiple as shown in the two views of Figure 4. Due to the large number of hits, the octopus shows a crowded situation hard to explore. When this happens, a message prompt invites the user to reduce the size of results, using the taxonomy filter above the octopus. In this example, selecting "Mammalia" and 
"Homo sapiens" restricts the map to 31 reported compositions matching 440 -linked glycan structures and 49 proteins carrying those glycans. Composition N1S1 (one $\mathrm{N}$-Acetylhexosamine, one $\mathrm{N}$-acetylneuraminic acid) describes the specific sialyl-Tn antigen structure, and it is connected to only one glycan structure in the database. In Figure 4A, on the right side of the octopus, GlyConnect shows this composition in relation with 13 proteins. The "Disease - Glycan" version of the octopus accessible in the toolbar above the map shows that the sialyl-GalNAc epitope (alternatively the sialyl-Tn antigen) appears in connection with several types of cancers like breast cancer, adenocarcinoma and leukaemia, suggesting an association with cancer tissues and/or body fluids. All currently reported compositions that contain the selected sialyl-GalNAc epitope, are listed in the centre list of the octopus. Selection of H1N1S1, for example, allows to further investigate the specificity of the connections. Figure 4B shows the results of this selection in the octopus. H1N1S1 matches two structures in the database. The two red links reveal that these matches contain the searched motif (sialyl-GalNAc epitope) with the addition of a galactose. These are two anomers, the core 1 and core 8 structures. Obviously, Figure 4A differs from Figure 4B in which 18 red connectors go through H1N1S1 to proteins. Only six protein links are shared between Figure $4 \mathrm{~A}$ and $\mathrm{B}$. Those seven (13-6) proteins specifically associated with the sialyl-GalNAc epitope can be further considered by clicking on the protein name. This example can be seen as the simulation of adding a galactose to an initial $O$-linked structure on the attachment to a glycoprotein.

GlyConnect usage can be further illustrated by considering a simple query for glycans matching selected structural properties. An example search for fucosylated, di-sialylated, bisecting $\mathrm{N}$-glycans, finds a unique connection between a protein and a structure shown in the octopus (Figure 5). Selecting the corresponding glycan record, the "Family" tab reveals several structures sharing the same composition. This collection can be filtered according to a tissue type (hemic system selected in the example) and submitted to the EpitopeXtractor. The latter lists all possible ligands that are found in the selected full structures and maps these ligands to the Glycoepitope network ${ }^{33}$. The result shows the concentration of glycoepitopes in a limited area of the network. 


\section{Navigation and visualisation to popularise glycobiology}

The Search interface as illustrated in the previous paragraphs is convenient to show the various views representing the molecular entities of Figure 1 as well as their characteristic properties in a given context. The query and the results are adapted for non-specialists in glycobiology yet obtaining useful information. In complement to Search, the Browse interface also supports users in getting a more global picture of the data. Each type of entity (as shown in Figure 1) can be listed and items easily filtered by names and each item is linked to its respective individual page whether a protein, a glycan structure, a composition or a glycosite. Furthermore, each type of contextual information such as taxon, tissue or disease can also be listed and filtered. In these cases, an individual page (e.g. tissue page) will show all glycans known to be expressed in that tissue according to collected and cited published references.

Irrespective of the starting point, GlyConnect browsing will navigate across the data as long as the user requests related information. For example, a composition page will refer to the matching glycan structures and further on to related tissues from where these glycans have been described. If available, information on any link(s) to a disease is provided in a dedicated page that will list all glycans known to be expressed in this disease. This list can then be related to the glycoproteins expressed. Glycosite and peptide data are currently more simply handled in the Browse interface where each peptide evidence for a site can be explored. In particular, despite the unreviewed status of large scale glycoproteomics datasets, many protein references were checked in order to limit redundancy and peptides were grouped when they obviously belong to the same protein with distinct accession numbers. A telling example is B4DPNO, an unreviewed UniProt entry matching Beta-2-glycoprotein 1 (APOH, P02749). B4DPNO with tryptic peptide YTTFEYPNTINFSCNTGFYLNGADSAK was listed in the identification data with three sialylated compositions attached (H5N4S1, H5N4S2, H5N4F2S1). It appeared that the corresponding site resulted from an S->N mutation that creates a new glycosite at Asn-107 in the original P02749 sequence: ${ }_{97}$ YTTFEYPNTISFSCNTGFYLNGADSAK ${ }_{123}$. This mutation is reported in P02749 and corresponds to variant dbSNP:rs1801692. Merging this information into the GlyConnect record of P02749 shows a summary of three defined sites matching the UniProt entry annotation confirmed at Asn-162 (5 structures, 1 composition), Asn-183 ( 1 composition) and Asn-253 ( 2 structures and 19 compositions) with the additional Asn-107 as a post-mutation event unreported so far. 
Note that one potential glycosite listed at Asn-193 in P02749 does not appear in GlyConnect. It may be due to the large size of the tryptic peptide in which this glycosylation site is embedded (>3200 Da) that subsequently defied detection within the mass spectrometric experiment. Alternatively, it may not be glycosylated. This example illustrates the potential for exploring site annotation in GlyConnect, which is not complete but may reveal previously unknown associations.

Glycosite and peptide information is particularly useful for other (glyco)proteomics studies of different biologies/diseases when data originates from glycoproteomics studies. This is now integrated in neXtProt where a link to GlyConnect site pages can be activated as evidence of glycosylation (when available). Likewise, GeneCards now includes GlyConnect data and ongoing collaboration with the developers of that resource will gradually determine the relevance of integrating more information. Efforts to cross-reference to other databases will continue to include glyco-information in a variety of bioinformatics resources and popularise the glycobiology viewpoint.

\section{A reference for MS-based glycoproteomics}

GlyConnect is designed to support glycoproteomics along with the tool collection of Glycomics@ExPASy. To begin with, it is already a trusted source of Unimod¹ with regards to listing masses of glycans that are possibly attached to proteins. The complete composition file of GlyConnect was shared with Unimod such that most of Unimod glycan mass entries now link to the corresponding GlyConnect composition page. From there, the user can navigate the related data as explained in the paragraphs above. In the same spirit, work is ongoing for cross-referencing ProSight ${ }^{43}$ and GlyConnect to support glycopeptide analysis in top down proteomics.

The major impact of integrating large scale glycoproteomics studies results in GlyConnect is the potential to substantiate UniProt annotation. Less than 4000 human proteins are annotated with strong evidence of $\mathrm{N}$-glycosylation. Though the consensus on the actual number of glycoproteins is not yet reached, the estimate of glycoforms ${ }^{1}$ is expected to exceed the magnitude of $10^{4}$. Despite the uneven level of reviewing, many $N$-glycosylation sites are predicted on the basis of the occurrence of the well-known sequon $N-X-S / T(X \neq P)$. This sequence-based prediction is annotated in UniProt entries as the result of "sequence 
analysis" and is associated the ECO:0000255 evidence tag as set by the Evidence Ontology (http://www.evidenceontology.org/).

In order to assess the potential for refining site annotation in UniProt through the accumulation of high throughput glycoproteomics data, we collected the set of human UniProt entries (release 2018_07) that contain only putative $\mathrm{N}$-glycosites tagged ECO:0000255. In this evaluation, entries with at least a PubMed reference associated to an $\mathrm{N}$-glycosite (ECO:0000269) were excluded. This resulted in a set of 3270 UniProt proteins out of which 289 were present in GlyConnect. We select here a few representative examples of the usefulness of GlyConnect in refining questions or raising potentially new questions from putting into perspective experimental data.

We first highlight the results regarding mammaglobulin-A (Q13296) and mammaglobulin-B (075556) reflecting experimental data extracted from the unreviewed reference ${ }^{21}$ that characterised intact glycopeptides in breast tumours. Corresponding UniProt entries are poorly informative regarding the function of these $57 \%$ identical proteins. Q13296 indicates expression in breast cancer but not 075556 . Sequence prediction spots two $N$-glycosites in mammaglobulin-A (Q13296) at Asn-53 and Asn-68. Asn-68 is conserved in mammaglobulin-B (075556) while at position 53 a N->D mutation disables glycosylation. As it turns out Asn-68 has only been detected as glycosylated in mammaglobulin-B (O75556) with two compositions matching oligomannose structures (H8N2, H9N2). However, the unique Asn-53 site in mammaglobulin-A (Q13296) is associated with 26 possible compositions, 11 of which are sialylated and 13 are fucosylated (eight are in common, i.e., both sialylated and fucoslyated). The identified tryptic glycopeptide was 20 amino acids long. Two compositions (H6N5F3S1 and H8N7S1) did not show a structure match in the database, while for another two compositions, single matched structures were found. Interestingly, of these single matches, the first one (H4N4F3) points to a structure containing two terminal Lewis $x$ glycoepitopes and the second (H6N6S3) has a bisecting GlcNAc. Although these compositions could also describe not yet reported/recorded structures in the database, the validation of these characteristics requires further investigation. The 22 remaining compositions all have multiple potential matches in the database though each can be associated with mutually exclusive features, such as oligomannose structures (7), triantennary (4), tetrantennary (2) and another three potentially with a bisecting GICNAC (H3N3, H3N3F1, H3N4). Note that mammaglobulin- 
A and B are alternatively spliced but splicing does not affect the glycosylation sites. The distinguishing glycosylation pattern of mammaglobulin-A that is known to be involved in breast cancer definitely provides substance for studying the pathological role of these protein glycoforms.

Another example where Glyconnect highlights novel areas of research is a pair of similar proteins with unknown function: Prominin-1 (043490) and Prominin-2 (Q8N271). While the above described mammaglobulins are 93 and 95 amino acid long, the length of prominins is 865 and 834, respectively. Consequently, significantly more sequon motifs can be expected. Surprisingly, Q8N271 reports only Asn-270 as a possible site (sequence-based prediction), while 043490 shows the prediction of eight $N$-sites (Asn-220, 274, 395, 414, 548, 580, 729, 730). As it turns out, only Asn-270/Asn-274 is conserved in the alignment of these two proteins (see supp. Figure 1). The glycoproteomics results of our source ${ }^{21}$ show selective $N$ glycosylation of two expected sites in prominin-1 (Asn-220, 274) and two unexpected sites (Asn-707, 725) in prominin-2. The conserved site is glycosylated in prominin-1 (Asn-274) but not in prominin-2 (Asn-270). Nevertheless, it is also possible that the tryptic glycopeptide containing Asn-270 was not detected due to its size (4849.899 Da without N-glycan). Nonetheless, these data indicate a tendency for $\mathrm{N}$-terminal $\mathrm{N}$-glycosylation in prominin-1 while the C-terminal part of prominin-2 is $\mathrm{N}$-glycosylated. Only one non-sialylated difucosylated composition is reported at Asn-220 on prominin-1, whereas twelve out of fifteen structures listed at Asn-274 are sialylated. The two $N$-sites of prominin-2 are associated with less specific compositions; Asn-707 contains three highly fucosylated structures (at least 3 fucoses) and Asn-725 only two sialylated compositions that are part of the list associated with Asn-274. Again, these details could become useful in the design of a functional study of prominins.

A last instructive example is Apolipoprotein F (Q13790) that is predicted to bear a single $\mathrm{N}$-glycosylated Asn-118 in the corresponding UniProt entry. Glycoproteomics studies confirm the occurrence of two compositions at Asn-118, one of which (N4H6F2) is highly specific in our database since it only points at structures carrying the Blood group A glycoepitope (of course, this may only reflect a bias in our data). The second composition (H5N4S2) is more common and matches multiple structural entries in the database. Interestingly, a second site 
not listed in UniProt Q13790 is suggested by experimental data at Asn-139 where the same disialylated composition (H5N4S2) is described.

Stored data of many other proteins, especially membrane and transmembrane, offer similar potential leads to hypothesize on the specific properties of $N$-glycosylation and its potential regulatory role.

\section{Seed assumptions on site assignment of glycans}

The accumulation of site-specific compositional data increasingly supports potential site assignment of previously identified structures that were characterised after they were released from the glycoproteins. For example, over the years the glycans on several abundant human proteins such as fibronectin or the epidermal growth factor receptor have been characterised in successive and independent MS-based experiments. The corresponding data populated the database with recurring and new glycan structures and/or compositions. Recent compositional inclusions from intact glycopeptide glycoproteomic experiments are all site-specific. As explained in the Material and Methods section, structures are mapped to compositions at the protein level so that defined structures listed in the "unknown site" category can be matched with site-specific compositions obtained from glycoproteomic data. Fibronectin at site Asn-1007 is reported to bear two defined released structures and nine compositions. The two structures match two of the separately reported compositions that also match two defined released structures which had been reported on fibronectin without known sites (grey border). A third defined structure reported without a known site(s) matches another composition (H5N4F1) on Asn-1007. No other structure listed in the "unknown site" section was found to match any of the six remaining compositions listed at this site. A sharper contrast regarding fucosylated structures is seen between Asn-528 and Asn-1236. Released structures with two fucoses have been reported without any site assignment and match compositions only seen on Asn-528. On Asn-1236 however, no fucosylated structure released from fibronectin is matched to any of the 18 listed compositions ( 8 fucosylated/10 nonfucoslyated). This trend would need to be confirmed but the likelihood of selective site glycosylation is indicated.

Following the same line of reasoning, data stored for the epidermal growth factor receptor (EGFR) reveals a definite preference for oligomannose glycans at Asn-352 and Asn-361 while 
Asn-413 appears to carry fucose-rich complex structures. Note that 42 of the $50(92 \%)$ reported structures at unknown sites on EGFR are fucosylated.

Overall, only 21 human $\mathrm{N}$-glycoproteins of the database are associated with both sitespecific compositions and defined released structures on unknown sites. An average of $62 \%$ of defined released structures reported on unknown sites could be assigned to a specific site by matching a composition (details are provided in Supp.Table I).

\section{Discussion}

Glycosylation is a major posttranslational modification (PTM) and its characterisation is essential in deciphering the combinatorial patterns of PTMs that convey information through 'PTM crosstalk' on the protein. This is considered as one of the next most important challenge in proteomics ${ }^{44}$. Prior to solving PTM crosstalk, it is necessary to qualify and quantify sitespecific modifications, in particular the highly heterogeneous and variable glycosylation.

GlyConnect is proposed as a solution to conquer this challenge and fill the existing gap for querying integrated data in glycobiology. As mentioned in the Introduction, the existing range of databases and tools that can support functional studies in glycobiology is limited, occurs on individual servers, is often not maintained and not integrated. Previously, some level of tool integration was achieved on a more chemically-oriented basis such as GLYCOSCIENCES.de ${ }^{6}$ or an array focussed basis in the CFG databases ${ }^{7}$. GlyConnect is designed to shift integration towards overall biological questions that involve glycoproteins. It needs to be acknowledged that this lack has also been due to that of high-throughput methodologies in glycomics and glycoproteomics. These fields have just started catching up to other -omics and generate reliable data that can now provide the data backbone of integrated tools as presented here with GlyConnect.

Mass spectrometry techniques associated to powerful bioinformatics tools have generated a significant portion of proteomic biological knowledge over the past two decades. Given the rapid development happening in the glycoproteomics space, these methodologies will also provide in-depth data on glycoproteins and their site-specific glycosylation patterns in health and disease. Tools such as GlyConnect enable the user to search, display and connect these complex data to other databases and provide interested scientists with an opportunity to find 
information that otherwise would be difficult to grasp. Nevertheless, still a lot of effort is necessary to develop workflows that enable structuring and standardising the data derived from the incoming flow of glycosylation-based data. Programs such as the MIRAGE initiative that define the standards for reporting of glyco-related data ${ }^{45}$ are one important step towards ensuring a minimum data and information standard.

Results presented above show that unearthed information in the numerous Excel spreadsheets submitted as supplementary material can already feed the annotation of glycoproteins. Our immediate target is therefore to build upon these types of resources and reinforce the early identified trends described in the Results section that emphasised that much more glyco-related information can be gained from existing knowledge if it is accessible. At the time of writing, straightforward literature search on PubMed (https://www.ncbi.nlm.nih.gov/pubmed/) reveals the existence of several datasets available for integration to feed the GlyConnect pipeline. We are aware that trends in the currently obtained data (human $\mathrm{N}$-glycans) induces bias that is reflecting the major interest in glycosylation in human diseases. This is an important and vital research, but alternative dedicated curation programmes that cover also other branches of life such as bacteria, other animals, plants, viruses and parasites need also to be investigated. The here presented tools, however, are designed to provide the backbone for such projects once increasing amounts of reliable data are available.

GlyConnect currently supports multiple types of comparison and associations. As shown in Figure 4, the octopus reveals the relations between glycoproteins and their individual glycan compositions and related structures. Moreover, the several versions of the octopus combine different concepts such as tissue and glycan, disease and glycan, etc, to highlight glycandependent connections that otherwise are impossible to gather from current highthroughput data outputs. GlyConnect supports scientists in making connections between compositions, glycoproteins and their changes when acquired by global glycoproteomics methods. If combined with parallel global glycomics studies that shine light on the specific structures present in a sample, educated structure predictions can be made from the compositional data. Although not yet fully exploited, Sankey graphs also bring another view on associations between entities depicted in Figure 1 . Work is in progress to refine and enhance this variety of views. A near future step of data integration will soon include 
UniLectin, a knowledgebase of carbohydrate-binding proteins that we co-develop. This will allow to display the potential set of lectins that can bind queried glyco-epitopes with the help of EpitopeXtractor and cross-links to the Glydin' epitope network. Despite the limitation of compositional data that will not always be specific for a peculiar glyco-epitope without orthogonal data, this does not limit the ability of GlyConnect to associate different data sources into an informative and visual output. Suggestions made throughout the results of the present paper have been made with caution and not stated as facts but as leads to refine questions or guide reasoning on the data.

\section{Conclusion}

GlyConnect is an evolving platform. It is the central engine of the Glycomics@ExPASy collection. Our near future plans include the definition of filters based on protein functional properties. We also plan to implement the evidence ontology (http://www.evidenceontology.org/) to qualify the reliability of information in the database. Substantial work is needed to cross-reference the Carbohydrate Active Enzyme database $\mathrm{CAZy}^{46}$ wisely and bring out glycan biosynthetic enzyme information in GlyConnect. Lastly, we are engaged in community-based efforts to promote the usage and automate the analysis of glycomics and glycoproteomics data. At this stage, we are moving towards data sharing via repositories $^{38}$ and co-developing software with several groups to ease graphic queries of glycan databases ${ }^{47}$. We are also testing the performance of the currently available glycoproteomics MS software with benchmark datasets HUPO Human Glycoproteomics Initiative (HGI: https://www.hupo.org/Glycoproteomics-(B/D-GPP)). GlyConnect is destined to become the curated and integrated component in this larger glycoinformatics picture.

\section{References}

(1) Aebersold, R.; Agar, J. N.; Amster, I. J.; Baker, M. S.; Bertozzi, C. R.; Boja, E. S.; Costello, C. E.; Cravatt, B. F.; Fenselau, C.; Garcia, B. A.; et al. How Many Human Proteoforms Are There? Nature Chemical Biology 2018, 14 (3), 206-214. https://doi.org/10.1038/nchembio.2576.

(2) Lisacek, F.; Mariethoz, J.; Alocci, D.; Rudd, P. M.; Abrahams, J. L.; Campbell, M. P.; Packer, N. H.; Ståhle, J.; Widmalm, G.; Mullen, E.; et al. Databases and Associated Tools for Glycomics and Glycoproteomics. In High-Throughput Glycomics and 
Glycoproteomics; Lauc, G., Wuhrer, M., Eds.; Springer New York: New York, NY, 2017; Vol. 1503, pp 235-264. https://doi.org/10.1007/978-1-4939-6493-2_18.

(3) Egorova, K. S.; Toukach, P. V. Glycoinformatics: Bridging Isolated Islands in the Sea of Data. Angewandte Chemie International Edition 2018. https://doi.org/10.1002/anie.201803576.

(4) Campbell, M. P.; Aoki-Kinoshita, K. F.; Lisacek, F.; York, W. S.; Packer, N. H. Glycoinformatics. In Essentials of Glycobiology; Varki, A., Cummings, R. D., Esko, J. D., Stanley, P., Hart, G. W., Aebi, M., Darvill, A. G., Kinoshita, T., Packer, N. H., Prestegard, J. H., et al., Eds.; Cold Spring Harbor Laboratory Press: Cold Spring Harbor (NY), 2015.

(5) Doubet, S.; Bock, K.; Smith, D.; Darvill, A.; Albersheim, P. The Complex Carbohydrate Structure Database. Trends Biochem. Sci. 1989, 14 (12), 475-477.

(6) Lutteke, T. GLYCOSCIENCES.de: An Internet Portal to Support Glycomics and Glycobiology Research. Glycobiology 2006, $16 \quad$ (5), 71R-81R. https://doi.org/10.1093/glycob/cwj049.

(7) Raman, R.; Venkataraman, M.; Ramakrishnan, S.; Lang, W.; Raguram, S.; Sasisekharan, R. Advancing Glycomics: Implementation Strategies at the Consortium for Functional $\begin{array}{lllll}\text { Glycomics. } & \text { Glycobiology 2006, } & \end{array}$ https://doi.org/10.1093/glycob/cwj080.

(8) Mariethoz, J.; alocci, D.; Gastaldello, A.; horlacher, O.; Gasteiger, E.; Rojas-Macias, M.; Karlsson, N. G.; Packer, N.; Lisacek, F. Glycomics@ExPASy: Bridging the Gap. Molecular \& Cellular Proteomics 2018, mcp.RA118.000799. https://doi.org/10.1074/mcp.RA118.000799.

(9) Varki, A.; Cummings, R. D.; Aebi, M.; Packer, N. H.; Seeberger, P. H.; Esko, J. D.; Stanley, P.; Hart, G.; Darvill, A.; Kinoshita, T.; et al. Symbol Nomenclature for Graphical Representations of Glycans. Glycobiology 2015, 25 (12), 1323-1324. https://doi.org/10.1093/glycob/cwv091.

(10) Varki, A.; Cummings, R. D.; Esko, J. D.; Freeze, H. H.; Stanley, P.; Marth, J. D.; Bertozzi, C. R.; Hart, G. W.; Etzler, M. E. Symbol Nomenclature for Glycan Representation. PROTEOMICS 2009, 9 (24), 5398-5399. https://doi.org/10.1002/pmic. 200900708.

(11) Joshi, H. J.; von der Lieth, C.-W.; Packer, N. H.; Wilkins, M. R. GlycoViewer: A Tool for Visual Summary and Comparative Analysis of the Glycome. Nucleic Acids Research 2010, 38 (suppl_2), W667-W670. https://doi.org/10.1093/nar/gkq446.

(12) Konishi, Y.; Aoki-Kinoshita, K. F. The GlycomeAtlas Tool for Visualizing and Querying Glycome Data. Bioinformatics 2012, 28 (21), 2849-2850. https://doi.org/10.1093/bioinformatics/bts516.

(13) Pérez, S.; Sarkar, A.; Rivet, A.; Breton, C.; Imberty, A. Glyco3D: A Portal for Structural Glycosciences. In Glycoinformatics; Lütteke, T., Frank, M., Eds.; Springer New York: New York, NY, 2015; Vol. 1273, pp 241-258. https://doi.org/10.1007/978-1-4939-23434_18.

(14) Sehnal, D.; Grant, O. C. Rapidly Display Glycan Symbols in 3D Structures: 3D-SNFG in LiteMol. Journal of Proteome 2018. https://doi.org/10.1021/acs.jproteome.8b00473.

(15) Woods, R. J. Predicting the Structures of Glycans, Glycoproteins, and Their Complexes. Chemical Reviews 2018, 118 (17), 8005-8024. https://doi.org/10.1021/acs.chemrev.8b00032.

(16) Joshi, H. J.; Jørgensen, A.; Schjoldager, K. T.; Halim, A.; Dworkin, L. A.; Steentoft, C.; Wandall, H. H.; Clausen, H.; Vakhrushev, S. Y. GlycoDomainViewer: A Bioinformatics 
Tool for Contextual Exploration of Glycoproteomes. Glycobiology 2018, 28 (3), 131136. https://doi.org/10.1093/glycob/cwx104.

(17) UniProt Consortium, T. UniProt: The Universal Protein Knowledgebase. Nucleic Acids Research 2018, 46 (5), 2699-2699. https://doi.org/10.1093/nar/gky092.

(18) Gaudet, P.; Argoud-Puy, G.; Cusin, I.; Duek, P.; Evalet, O.; Gateau, A.; Gleizes, A.; Pereira, M.; Zahn-Zabal, M.; Zwahlen, C.; et al. NeXtProt: Organizing Protein Knowledge in the Context of Human Proteome Projects. Journal of Proteome Research 2013, 12 (1), 293298. https://doi.org/10.1021/pr300830v.

(19) Safran, M.; Dalah, I.; Alexander, J.; Rosen, N.; Iny Stein, T.; Shmoish, M.; Nativ, N.; Bahir, I.; Doniger, T.; Krug, H.; et al. GeneCards Version 3: The Human Gene Integrator. Database 2010, 2010 (0), baq020-baq020. https://doi.org/10.1093/database/baq020.

(20) Cooper, C. A.; Joshi, H. J.; Harrison, M. J.; Wilkins, M. R.; Packer, N. H. GlycoSuiteDB: A Curated Relational Database of Glycoprotein Glycan Structures and Their Biological Sources. 2003 Update. Nucleic Acids Res. 2003, 31 (1), 511-513.

(21) Hu, Y.; Shah, P.; Clark, D. J.; Ao, M.; Zhang, H. Reanalysis of Global Proteomic and Phosphoproteomic Data Identified a Large Number of Glycopeptides. Analytical Chemistry 2018, 90 (13), 8065-8071. https://doi.org/10.1021/acs.analchem.8b01137.

(22) Bollineni, R. C.; Koehler, C. J.; Gislefoss, R. E.; Anonsen, J. H.; Thiede, B. Large-Scale Intact Glycopeptide Identification by Mascot Database Search. Scientific Reports 2018, 8 (1). https://doi.org/10.1038/s41598-018-20331-2.

(23) Darwen, H.; Date, C. J.; Fagin, R. A Normal Form for Preventing Redundant Tuples in Relational Databases. In Proceedings of the 15th International Conference on Database Theory - ICDT '12; ACM Press: Berlin, Germany, 2012; p 114. https://doi.org/10.1145/2274576.2274589.

(24) Kent, W. A Simple Guide to Five Normal Forms in Relational Database Theory. Communications of the ACM 1983, $26 \quad$ (2), 120-125. https://doi.org/10.1145/358024.358054.

(25) Tiemeyer, M.; Aoki, K.; Paulson, J.; Cummings, R. D.; York, W. S.; Karlsson, N. G.; Lisacek, F.; Packer, N. H.; Campbell, M. P.; Aoki, N. P.; et al. GlyTouCan: An Accessible Glycan Structure Repository. Glycobiology 2017, 1-5. https://doi.org/10.1093/glycob/cwx066.

(26) Campbell, M. P.; Ranzinger, R.; Lütteke, T.; Mariethoz, J.; Hayes, C. A.; Zhang, J.; Akune, Y.; Aoki-Kinoshita, K. F.; Damerell, D.; Carta, G.; et al. Toolboxes for a Standardised and Systematic Study of Glycans. BMC Bioinformatics 2014, 15 (Suppl 1), S9. https://doi.org/10.1186/1471-2105-15-S1-S9.

(27) Herget, S.; Ranzinger, R.; Maass, K.; Lieth, C.-W. v. d. GlycoCT-a Unifying Sequence Format for Carbohydrates. Carbohydrate Research 2008, 343 (12), 2162-2171. https://doi.org/10.1016/j.carres.2008.03.011.

(28) Essentials of Glycobiology, 2nd ed.; Varki, A., Ed.; Cold Spring Harbor Laboratory Press: Cold Spring Harbor, N.Y, 2009.

(29) Sharon, N. Nomenclature of Glycoproteins, Glycopeptides and Peptidoglycans (Recommendations 1985). Pure and Applied Chemistry 1988, 60 (9), 1389-1394. https://doi.org/10.1351/pac198860091389.

(30) Harvey, D. J.; Merry, A. H.; Royle, L.; Campbell, M. P.; Dwek, R. A.; Rudd, P. M. Proposal for a Standard System for Drawing Structural Diagrams of $\mathrm{N}$ - and O-Linked Carbohydrates and Related Compounds. Proteomics 2009, 9 (15), 3796-3801. https://doi.org/10.1002/pmic.200900096. 
(31) Mariethoz, J.; Khatib, K.; Alocci, D.; Campbell, M. P.; Karlsson, N. G.; Packer, N. H.; Mullen, E. H.; Lisacek, F. SugarBindDB, a Resource of Glycan-Mediated Host-Pathogen Interactions. Nucleic Acids Research 2016, 44 (D1), D1243-D1250. https://doi.org/10.1093/nar/gkv1247.

(32) Gastaldello, A.; Alocci, D.; Baeriswyl, J.-L.; Mariethoz, J.; Lisacek, F. GlycoSiteAlign: Glycosite Alignment Based on Glycan Structure. Journal of Proteome Research 2016, 15 (10), 3916-3928. https://doi.org/10.1021/acs.jproteome.6b00481.

(33) Alocci, D.; Ghraichy, M.; Barletta, E.; Gastaldello, A.; Mariethoz, J.; Lisacek, F. Understanding the Glycome: An Interactive View of Glycosylation from Glycocompositions to Glycoepitopes. Glycobiology 2018, 28 (6), 349-362. https://doi.org/10.1093/glycob/cwy019.

(34) Ceroni, A.; Dell, A.; Haslam, S. M. The GlycanBuilder: A Fast, Intuitive and Flexible Software Tool for Building and Displaying Glycan Structures. Source Code for Biology and Medicine 2007, 2 (1), 3. https://doi.org/10.1186/1751-0473-2-3.

(35) Creasy, D. M.; Cottrell, J. S. Unimod: Protein Modifications for Mass Spectrometry. PROTEOMICS 2004, 4 (6), 1534-1536. https://doi.org/10.1002/pmic. 200300744.

(36) Clerc, F.; Reiding, K. R.; Jansen, B. C.; Kammeijer, G. S. M.; Bondt, A.; Wuhrer, M. Human Plasma Protein N-Glycosylation. Glycoconjugate Journal 2016, 33 (3), 309-343. https://doi.org/10.1007/s10719-015-9626-2.

(37) Sehnal, D.; Deshpande, M.; Vařeková, R. S.; Mir, S.; Berka, K.; Midlik, A.; Pravda, L.; Velankar, S.; Koča, J. LiteMol Suite: Interactive Web-Based Visualization of Large-Scale Macromolecular Structure Data. Nature Methods 2017, 14 (12), 1121-1122. https://doi.org/10.1038/nmeth.4499.

(38) Rojas-Macias, M. A.; Mariethoz, J.; Andersson, P.; Jin, C.; Venkatakrishnan, V.; Aoki, N. P.; Shinmachi, D.; Ashwood, C.; Madunic, K.; Zhang, T.; et al. E-Workflow for Recording of Glycomic Mass Spectrometric Data in Compliance with Reporting Guidelines. 2018. https://doi.org/10.1101/401141.

(39) Federhen, S. The NCBI Taxonomy Database. Nucleic Acids Research 2012, 40 (D1), D136-D143. https://doi.org/10.1093/nar/gkr1178.

(40) Gremse, M.; Chang, A.; Schomburg, I.; Grote, A.; Scheer, M.; Ebeling, C.; Schomburg, D. The BRENDA Tissue Ontology (BTO): The First All-Integrating Ontology of All Organisms for Enzyme Sources. Nucleic Acids Research 2011, 39 (Database), D507-D513. https://doi.org/10.1093/nar/gkq968.

(41) Mungall, C. J.; Torniai, C.; Gkoutos, G. V.; Lewis, S. E.; Haendel, M. A. Uberon, an Integrative Multi-Species Anatomy Ontology. Genome Biology 2012, 13 (1), R5. https://doi.org/10.1186/gb-2012-13-1-r5.

(42) Schriml, L. M.; Arze, C.; Nadendla, S.; Chang, Y.-W. W.; Mazaitis, M.; Felix, V.; Feng, G.; Kibbe, W. A. Disease Ontology: A Backbone for Disease Semantic Integration. Nucleic Acids Research 2012, 40 (D1), D940-D946. https://doi.org/10.1093/nar/gkr972.

(43) Fellers, R. T.; Greer, J. B.; Early, B. P.; Yu, X.; LeDuc, R. D.; Kelleher, N. L.; Thomas, P. M. ProSight Lite: Graphical Software to Analyze Top-down Mass Spectrometry Data. PROTEOMICS 2015, 15 (7), 1235-1238. https://doi.org/10.1002/pmic.201400313.

(44) Venne, A. S.; Kollipara, L.; Zahedi, R. P. The next Level of Complexity: Crosstalk of Posttranslational Modifications. PROTEOMICS 2014, 14 (4-5), 513-524. https://doi.org/10.1002/pmic.201300344.

(45) Kolarich, D.; Rapp, E.; Struwe, W. B.; Haslam, S. M.; Zaia, J.; McBride, R.; Agravat, S.; Campbell, M. P.; Kato, M.; Ranzinger, R.; et al. The Minimum Information Required for 
a Glycomics Experiment (MIRAGE) Project: Improving the Standards for Reporting Mass-Spectrometry-Based Glycoanalytic Data. Molecular \& Cellular Proteomics 2013, 12 (4), 991-995. https://doi.org/10.1074/mcp.0112.026492.

(46) Lombard, V.; Golaconda Ramulu, H.; Drula, E.; Coutinho, P. M.; Henrissat, B. The Carbohydrate-Active Enzymes Database (CAZy) in 2013. Nucleic Acids Res. 2014, 42 (Database issue), D490-495. https://doi.org/10.1093/nar/gkt1178.

(47) Alocci, D.; Suchánková, P.; Costa, R.; Hory, N.; Mariethoz, J.; Vařeková, R.; Toukach, P.; Lisacek, F. SugarSketcher: Quick and Intuitive Online Glycan Drawing. Molecules 2018, 23 (12), 3206. https://doi.org/10.3390/molecules23123206.

\section{Author Contributions}

Conceptualization, D.A., J.M. and F.L.; methodology, D.A., J.M. and F.L.; software, D.A., A.G., and J.M.; validation, N.G.K, D.K., N.H.P., E.G. and F.L.; writing-original draft preparation, D.A. and F.L.; writing-review and editing, all.; supervision, N.H.P and F.L.; funding acquisition, N.H.K and F.L

\section{Funding}

This work is supported by the Swiss Federal Government through the State Secretariat for Education, Research and Innovation (SERI). The ExPASy portal is maintained by the web team of the Swiss Institute of Bioinformatics and hosted at the Vital-IT Competency Center. Part of the tools described above were developed with the support of the European Union FP7 Innovative Training Network [ITN 316929]

\section{Acknowledgements}

We thank the neXProt and GeneCards teams for constructive interactions. 


\section{Tables}

\section{Table I}

Cross-references in GlyConnect

\begin{tabular}{|l|l|l|}
\hline Cross-reference & Resource & URL \\
\hline Glycan & GlyTouCan & glytoucan.org \\
\hline Protein & UniProtKB & uniprot.org \\
\hline & neXtProt & nextprot.org \\
\hline Gene & UniLectin & unilectin.eu \\
\hline 3D Structures & GeneCards & genecards.org \\
\hline Reference & Protein Data Bank & ebi.ac.uk/pdbe/ \\
\hline (via Litemol) & PubMed & ncbi.nlm.nih.gov/pubmed \\
\hline Taxonomy & DOI & doi.org \\
\hline Disease & NCBI Taxonomy & ncbi.nlm.nih.gov/taxonomy \\
\hline & Disease Ontology & disease-ontology.org \\
\hline
\end{tabular}




\section{Table Ila}

Glycan structural properties used in GlyConnect

\begin{tabular}{|c|c|c|}
\hline Category & Property type & Property name \\
\hline \multirow[t]{4}{*}{ Compositional } & \multirow[t]{4}{*}{ Fucosylation } & Fucosylated \\
\hline & & Core-fucosylated \\
\hline & & Non-fucosylated \\
\hline & & Non-core-fucosylated \\
\hline \multirow[t]{2}{*}{ Compositional } & \multirow[t]{2}{*}{ Galactosylation } & Galactosylated \\
\hline & & Non-galactosylated \\
\hline \multirow[t]{5}{*}{ Compositional } & \multirow[t]{5}{*}{ Sialylation } & Sialylated \\
\hline & & Non-sialylated \\
\hline & & Mono-sialylated \\
\hline & & Di-sialylated \\
\hline & & Over-two-sialylated \\
\hline \multirow[t]{4}{*}{ Compositional } & \multirow[t]{4}{*}{ Xylosylation } & Xylosylated \\
\hline & & Core-xylosylated \\
\hline & & Non-xylosylated \\
\hline & & Non-core-xylosylated \\
\hline \multirow[t]{4}{*}{ Structural } & \multirow[t]{4}{*}{ Antenna } & Bi-antennary \\
\hline & & Tri-antennary \\
\hline & & Tetra-antennary \\
\hline & & Over-tetra-antennary \\
\hline \multirow[t]{2}{*}{ Structural } & \multirow[t]{2}{*}{ Bisection } & Bisecting \\
\hline & & Non-bisecting \\
\hline \multirow[t]{2}{*}{ Structural } & \multirow[t]{2}{*}{ Truncation } & Truncated \\
\hline & & Non-truncated \\
\hline
\end{tabular}


Table Ilb:

Glycan cores in the database

\begin{tabular}{|c|c|}
\hline Glycan type & Core name \\
\hline Free & Complex \\
\hline Free & Lactose \\
\hline Free & No-core \\
\hline Free & None \\
\hline Free & Truncated \\
\hline$N$-Linked & Complex \\
\hline$N$-Linked & High-Mannose \\
\hline$N$-Linked & Hybrid \\
\hline$N$-Linked & No-core \\
\hline$N$-Linked & Pauci-Mannose \\
\hline$N$-Linked & Truncated \\
\hline$N$-Linked & Undefined core \\
\hline$N$-Linked/O-Linked & No-core \\
\hline$N$-Linked/O-Linked & Truncated If $N$-Linked \\
\hline$O$-Linked & Core 0 \\
\hline$O$-Linked & Core 1 \\
\hline O-Linked & Core 10 \\
\hline O-Linked & Core 11 \\
\hline O-Linked & Core 12 \\
\hline$O$-Linked & Core 13 \\
\hline O-Linked & Core 14 \\
\hline O-Linked & Core 2 \\
\hline O-Linked & Core 3 \\
\hline O-Linked & Core 4 \\
\hline O-Linked & Core 5 \\
\hline O-Linked & Core 6 \\
\hline O-Linked & Core 7 \\
\hline O-Linked & Core 8 \\
\hline
\end{tabular}




\begin{tabular}{|l|l|}
\hline O-Linked & Core 9 \\
\hline O-Linked & No-core \\
\hline O-Linked & Undefined core \\
\hline O-Linked/C-Linked & No-core \\
\hline Undefined type & No-core \\
\hline Undefined type & Undefined core \\
\hline
\end{tabular}

Table III

Notation of monosaccharides

\begin{tabular}{|c|l|l|l|}
\hline Full name & Short name & Encoding & Symbol \\
\hline Hexose & Hex & H & circle \\
\hline N-Acetylhexosamine & HexNAc & $\mathrm{N}$ & square \\
\hline Fucose & Fuc & $\mathrm{F}$ & triangle \\
\hline $\begin{array}{c}\text { N-Glycolyl-Neuraminic acid } \\
\text { (Sialic acid) }\end{array}$ & NeuGc & $\mathrm{G}$ & $\begin{array}{c}\text { diamond } \\
\text { (purple) }\end{array}$ \\
\hline $\begin{array}{c}\text { N-Acetyl-Neuraminic Acid } \\
\text { (Sialic acid) }\end{array}$ & NeuAc & $\mathrm{S}$ & $\begin{array}{c}\text { diamond } \\
\text { (light blue) }\end{array}$ \\
\hline \begin{tabular}{c} 
Sulphate \\
\hline
\end{tabular} & S & $\mathrm{S}$ & $\mathrm{S}$ \\
\hline
\end{tabular}




\section{Figures}

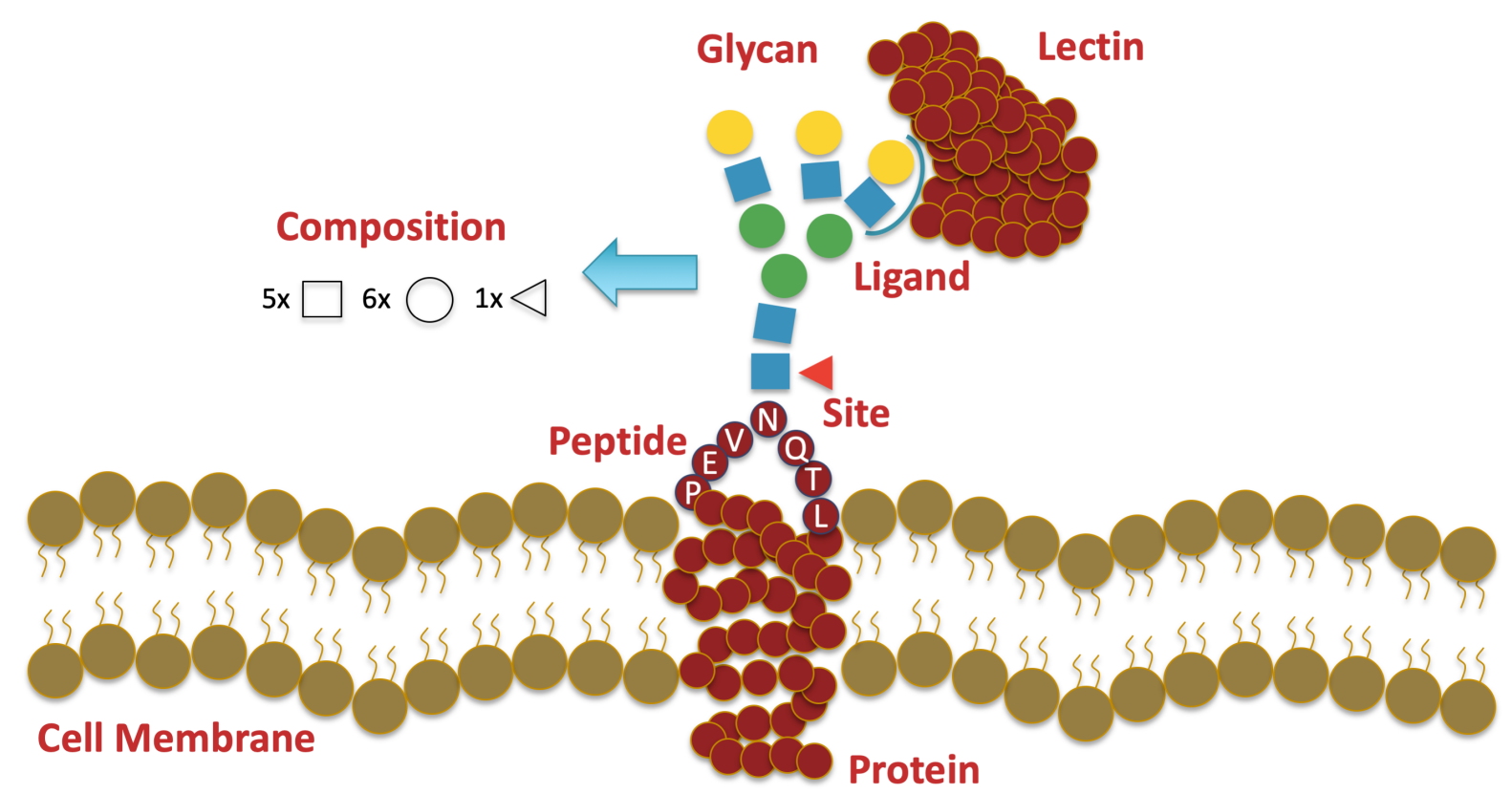

\section{$\underline{\text { Figure } 1}$}

Depiction of the cell membrane harbouring a glycoprotein. The cell membrane accommodates a transmembrane protein which is decorated by a glycan structure attached on the asparagine residue $(\mathrm{N})$ part of the characteristic sequon NQT; $N$ is the glyco(sylation) site. Additionally, a subregion of the glycan structure, called ligand or glyco-epitope or glycan determinant, interacts with a glycan-binding protein or lectin. Peptide and composition are notions related to the experimental techniques such as mass spectrometry used to identify intact glycoproteins. In glycoproteomics, proteins are digested into peptides to be analysed, and attached glycans are usually elucidated as compositions, a list of all the building blocks that compose a structure. 


\section{Inside GlyConnect}

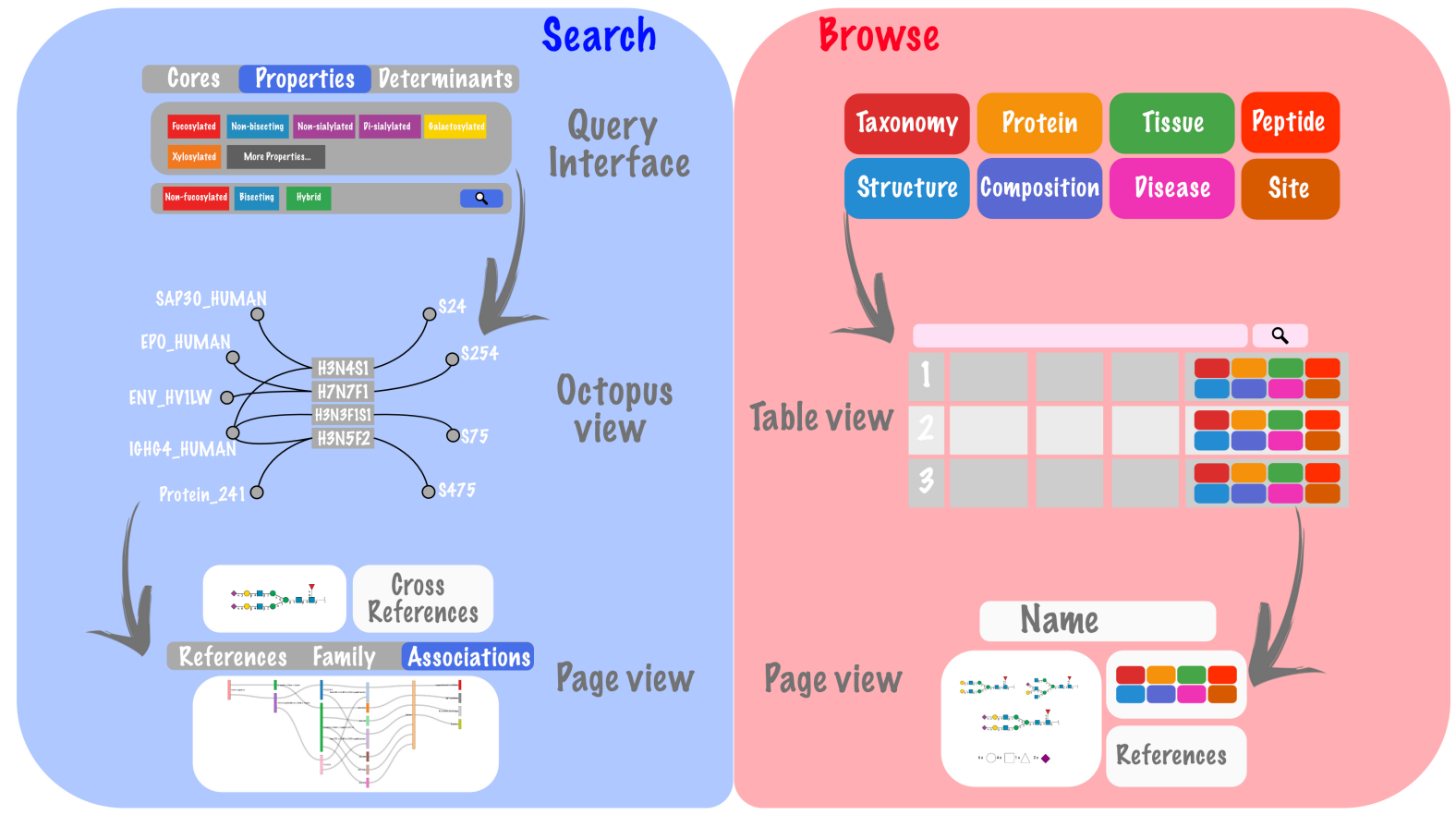

Figure 2

GlyConnect Overview. This figure summarises the content of Glyconnect while illustrating the two interfaces available in GlyConnect: Search and Browse. The Search interface proposes an intuitive query builder for exploring glycans and proteins. Results are shown in a conceptual map familiarly called octopus for its shape. The Browse interface displays data in lists of items and proposes a global view of the data. GlyConnect includes data reviewed by experts (manual annotation) and unreviewed data from high-throughput studies. 


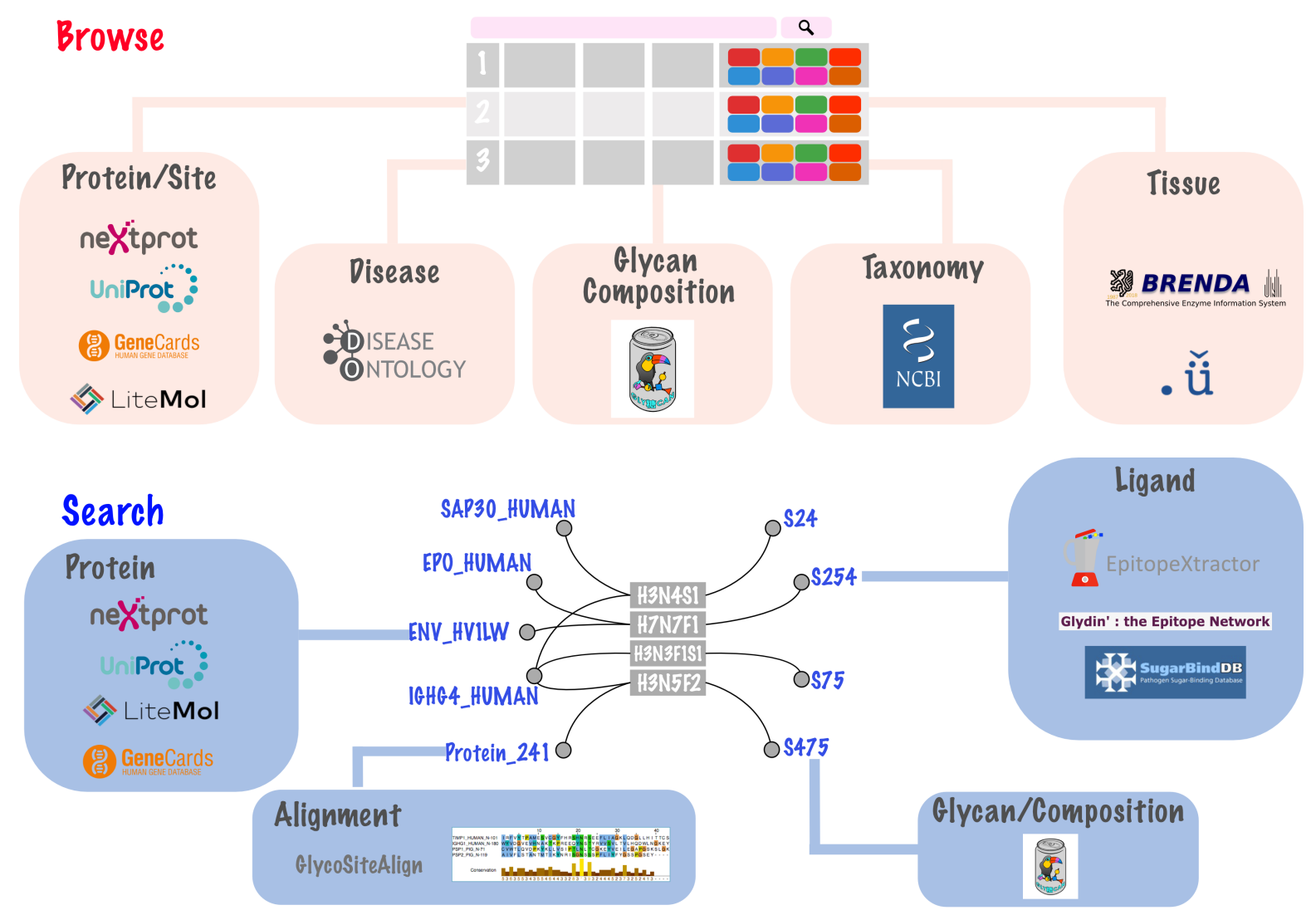

\section{Figure 3}

Connectivity of GlyConnect. This figure describes in finer details the two interfaces of GlyConnect featuring in Figure 2 focusing on cross-references and in-house tools integration. The information stored in the database, can be explored using both the Browse and Search interfaces. The Browse interface is composed of eight types of pages: Taxonomy, Tissue, Glycan, Composition, Protein, Peptide, Site and Disease. All pages have the same look and feel but each one is cross-referenced with different sources according to the content (i.e. disease page is connected to disease ontology). The Search interface proposes an interactive query builder and a conceptual map, called octopus, to show the results. From the octopus, it is possible to run GlycoSiteAlign, which performs the alignment of amino acid sequences based on glycan features. Additionally, the search interface has dedicated protein and glycan pages and uses the browser pages for Taxonomy, Tissue and Disease. These specific glycan and protein pages are reachable only from the octopus and they have been integrated with external tools. Selected protein pages have a link to a PDB 3D-viewer, called LiteMol, which displays the glycoprotein together with sugars in SNFG 3D. Sugar pages are connected with 
EpitopeXtractor which extracts ligands from a collection of structures based on a list of almost 600 glycan epitopes. The list of extracted epitopes can be plotted on Glydin', a network visualisation of glycan epitopes. Some of these epitopes are cross-referenced to SugarBindDB, a database on host-pathogen interactions mediated by glycans. All GlyConnect data is supported by a PubMed reference. 

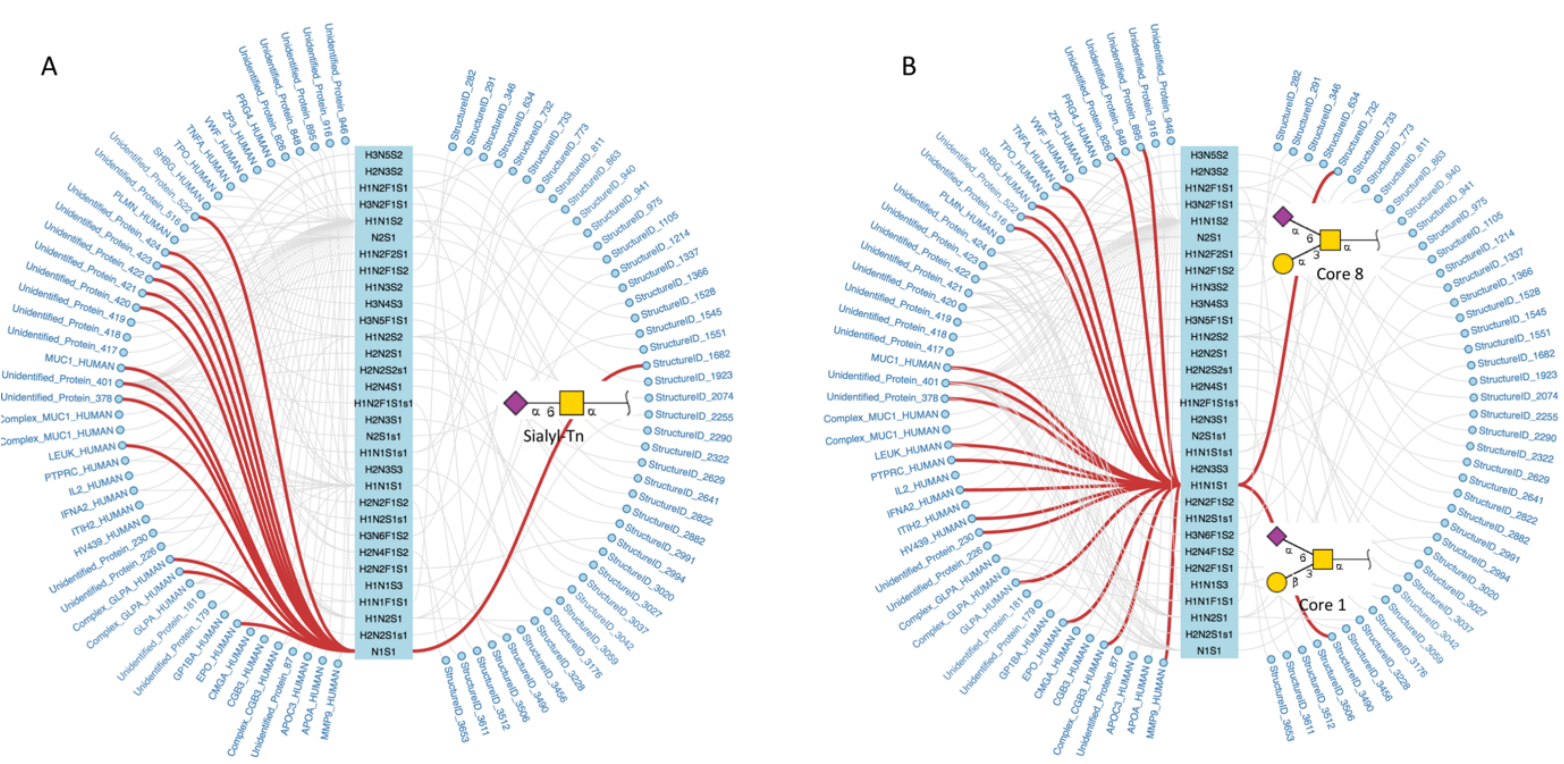

Figure 4.

Octopus result map. The figure shows the octopus result map generated by querying all $O$ linked glycans which contain Sialyl-Tn antigen in Homo sapiens. (A) The composition N1S1 is the exact composition of the Sialyl-Tn antigen and the octopus shows connections highlighted in red. On the structure side (right) only the Sialyl-Tn antigen appears and on the protein side (left) 13 proteins are connected. (B) The composition H1N1S1 is the exact composition of core 1 and core 80 -glycans which differ by one galactose from the Sialyl-Tn antigen motif/ sialylGaINAc and the octopus shows corresponding connections highlighted in red. On the structure side, only core 1 and core 8 appear and on the protein side (left) 18 proteins are connected, six of which are common with those shown for the Sialyl-Tn antigen motif/ sialylGalNAc. 


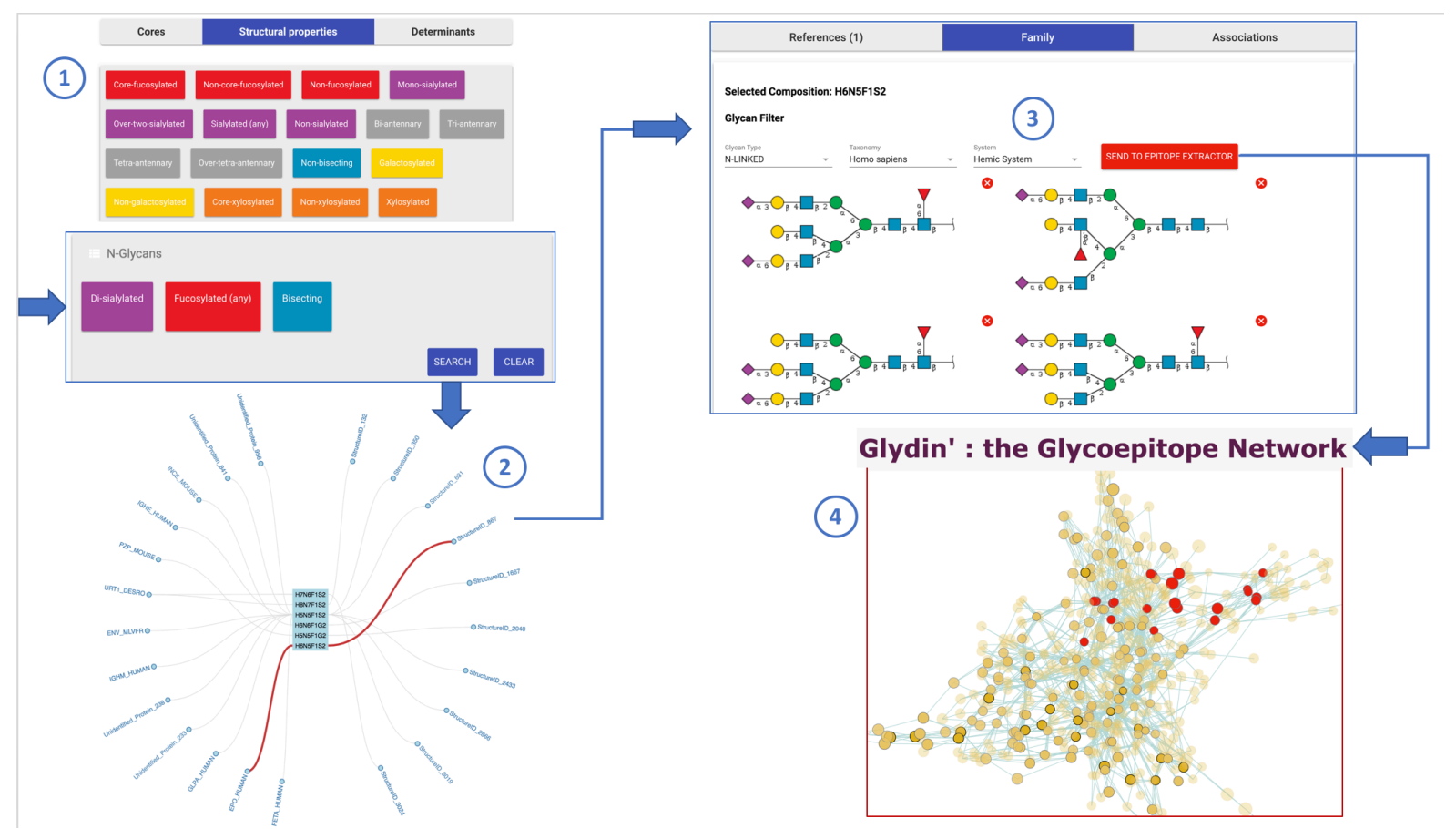

Figure 5.

Integrated results. After launching an $\mathrm{N}$-glycan search based on structural criteria which are here "fucosylated", "di-sialylated", "bisecting" (circled 1), the results are displayed in the octopus (circled 2). One of the compositions matching the query shows a unique association between a glycoprotein and an $\mathrm{N}$-glycan structure. Opening the corresponding glycan structure page (circled 3) and selecting the "Family" tab displays structural variations recorded in the database (same composition but different architectures). These can be (de)selected to become the input of EpitopeXtractor that generates the possible ligands in those structures as defined in a large collection of known ligands/glycoepitopes. In this example, the structures were filtered by tissue expression. The displayed structures are found in human blood. Then, the results of EpitopeXtractor are mapped on the "Glycoepitope Network" (circled 4). Ligands output by EpitopeXtractor are shown as red nodes in the network. In this example they are clearly co-localised. This information can for instance, guide a glycan array experiment. Note that blue arrows indicate the steps of processing. 